\title{
Psychometric Assessment of Scales for Measuring Loneliness and Social Isolation: An Analysis of the Household, Income and Labour Dynamics in Australia (HILDA) Survey
}

Karine Manera ( $\square$ karine.manera@sydney.edu.au )

The University of Sydney https://orcid.org/0000-0002-0552-6074

\section{Ben Smith}

The University of Sydney

\section{Katherine Owen}

The University of Sydney

\section{Philayrath Phongsavan}

The University of Sydney

Michelle Lim

Swinburne University of Technology

\section{Research}

Keywords: Loneliness, social isolation, structural equation modeling, factor analysis, reliability

Posted Date: October 11th, 2021

DOI: https://doi.org/10.21203/rs.3.rs-944678/v1

License: (1) This work is licensed under a Creative Commons Attribution 4.0 International License. Read Full License 


\section{Abstract}

Background: Loneliness and social isolation are increasingly recognised as global public health threats, meaning that reliable and valid measures are needed to monitor these conditions at a population level. We aimed to determine if robust and practical scales could be derived for conditions such as loneliness and social isolation using items from a national survey.

Methods: We conducted psychometric analyses of ten items in two waves of the Household, Income and Labour Dynamics in Australia Survey, which included over 15,000 participants. We used the Hull method, exploratory structural equation modelling, and multidimensional item response theory analysis in a calibration sample to determine the number of factors and items within each factor. We cross-validated the factor structure using confirmatory factor analysis in a validation sample. We assessed construct validity by comparing the resulting sub-scales with measures for psychological distress and mental well-being.

Results: Calibration and cross-validation consistently revealed a three-factor model, with sub-scales reflecting constructs of loneliness and social isolation. Sub-scales showed high reliability and measurement invariance across waves, gender, and age. Construct validity was supported by significant correlations between the sub-scales and measures of psychological distress and mental health. Individuals who met threshold criteria for loneliness and social isolation had consistently greater odds of being psychologically distressed and having poor mental health than those who did not.

Conclusions: These derived scales provide robust and practical measures of loneliness and social isolation for population-based research.

\section{Introduction}

Loneliness and social isolation are increasingly recognised as global public health threats. Loneliness is characterised as the negative subjective appraisal of social relationships, elicited by feeling like one's social relationships fail to adequately meet one's social needs [1]. Social isolation is the objective absence of social interactions, often measured in terms of the size and frequency of contacts [2]. Studies have shown that loneliness and social isolation are significant contributors to higher rates of premature mortality, with increased risks of $26 \%$ and $29 \%$, respectively, compared with people who are not lonely or isolated [3].

Given the growing body of evidence that loneliness and social isolation are important but neglected public health risk factors [4], there is a strong need to develop psychometrically validated and sound measures that can be used to identify and monitor the prevalence, distribution and trends in these conditions at the population level. Using reliable and valid instruments is therefore paramount for understanding the extent of loneliness and social isolation, and their subsequent impact on health and health-related behaviours. However, measuring the prevalence and distribution of these conditions in populations has been historically difficult. Population-based surveys, conducted as either telephone interviews or self-complete questionnaires, commonly involve the collection of a whole sweep of measures and can be lengthy and laborious to complete. Respondents are vulnerable to survey fatigue resulting in inaccuracies in responses to questions, hence brief measures are often favoured to ensure that respondents remain engaged [5]. For the assessment of constructs such as loneliness or social isolation, the use of longer scales (i.e., greater than 10 items) has been recommended for individual-level assessment in clinical settings, while shorter scales are found to be particularly beneficial for providing broad, population-level estimates, and for guiding public health and policy responses [6].

Although loneliness and social isolation are widely used constructs, different understandings about their nature and attributes are reflected in the variety of short- and long-form measures of these conditions being used in clinical and population surveys $[7,8]$. Commonly used measures of loneliness include the 20-item Revised UCLA Loneliness Scale [9], the 11-item De Jong Gierveld Ioneliness scale [10] and in social isolation, the 10-item Lubben Social Network Scale [11], and Berkman Social Network Index [12]. Shortened versions of these scales have been developed for use in large surveys, however these have all been developed and tested in surveys of older adults and infrequently used in surveys that are representative of the general population [13-15].

The Household Income and Labour Dynamics in Australia (HILDA) survey is a nationally representative panel study of over 9,000 Australian households conducted annually, with wave 1 of the survey starting in 2001 [16]. The HILDA survey follows participants from age 15 years onwards and collects data on household and family relationships, income, employment, health, and education. While the HILDA self-completion questionnaire includes a number of items concerning social interactions and social support, previous estimates of the prevalence of loneliness among study participants have been derived from responses to the single-item 
statement "I often feel very lonely" [17]. This is consistent with several other large surveys that have used single-item measures for Ioneliness $[18,19]$. While efficient, these single-item measures may be subjected to response bias, as loneliness is well-known to be associated with stigma, social desirability bias and gender bias (i.e., men are less likely to report loneliness than women [20-22]. The finding that individuals respond differently to one-item direct measures such as Are you lonely? as opposed to Would you like company? [23] also highlights the challenges in measuring this construct.

In light of these complexities, it is optimal to measure loneliness and other dimensions of social support and relationships using multiple items as opposed to a single item [24]. We aimed to undertake psychometric analysis of items examining social support in the HILDA survey, to determine if robust and practical scales could be derived for conditions such as loneliness and social isolation within this large population study.

\section{Methods}

\section{Participants}

Data used in this study were collected from the HILDA survey. In this cohort study a number of household-level and person-level questionnaires are completed for each household. The present study uses data from the self-completion questionnaire (herein called 'HILDA questionnaire'), which collects person-level data and is undertaken by individuals aged 15 years or older in each participating household. Approximately 15,000 individuals respond to the HILDA questionnaire each year. In this study we included individuals who completed the questionnaire in waves 17 (2017) and 19 (2019), which were chosen to provide a contemporary sample and to assess the consistency of psychometric properties across time.

\section{Measures}

The HILDA self-completion questionnaire collects comprehensive information about a person's general health and well-being, lifestyle and living situation, finances, work, and parenting. This instrument includes 10 items in which participants are required to describe and appraise their social support, by responding on a 7-point Likert scale from 1 (strongly disagree) to 7 (strongly agree) to a sequence of statements. Seven of the 10 items come from Henderson, Duncan-Jones [25] and three from Marshall and Barnett [26]. In previous analyses of the HILDA survey data these items have been used as a single scale for measuring social support and social networks [27, 28], but to our knowledge this scale has not been validated. A list of the 10 items is included in Supplementary Figure 1.

Each wave of the HILDA questionnaire includes the 36-item Short Form Survey (SF-36) [29]. In this study, we used the Mental Component Summary (MCS) from the SF-36 as a measure of mental health status for validation purposes. The full SF-36 survey instrument and guide to computing the MCS are freely available online. In brief, the MCS is calculated in a three-step process involving: i) standardising each of the eight SF-36 health domains using a Z-score transformation of means and standard deviations from the 1995 Australian National Health Survey (NHS) [30]; ii) aggregating Z-scores using coefficients from the NHS as weights; and iii) transforming scores to have a mean of 50 and a standard deviation of 10 . Individuals with MCS scores of 42 or below, which has proven to be a cut-point indicative of clinical depression, are classified as having poor mental health [31].

The other measure used for validation purposes was the Kessler Psychological Distress Scale (K10), which has been included in every second wave of the HILDA survey from 2007 [32]. The K10 provides a measure of non-specific psychological distress based on questions about negative emotional states experienced in the past 4-week period. Scores on the K10 range from 10 to 50 with higher scores indicating higher levels of psychological distress. Designated cut-off scores have previously been assigned as low to moderate (10-21) and high to very high (22-50) levels of psychological distress. In this study, we assigned those scoring 22 or higher on the K10 as having psychological distress [32-34].

To include a contemporary sample of participants who have data for the 10 social support items, SF-36 and K10, we included all individuals who completed the self-completion questionnaire in waves 17 and 19.

\section{Statistical analysis}


We calculated descriptive statistics, including frequencies and proportions, for individuals across the two waves. For each of the two waves we randomly allocated participants into a calibration sample (wave $17 n=7,869$; wave $19 n=7,549$ ) and validation sample (wave $17 n=7,549$; wave $19 n=7,705$ ). Using the calibration samples, we identified the number of factors and items within each factor based on results from the Hull method, exploratory structural equation modelling, and multidimensional item response theory analysis. Negatively worded items (items 1, 2, 4, 5 and 7) were reverse coded for all analyses so that a high value indicates the same type of response on every item. We evaluated the resulting factor structure using confirmatory factor analysis on the validation samples. Analyses were conducted using R software version 4.0.4, SPSS version 25 and flexMIRT version 3.0.

\section{Factor Structure and Item Selection}

The Hull method identifies a model with an optimal balance of model fit and number of parameters [35]. This method (HULL function in package 'EFAtools') was used to determine the number of factors to retain, with principal axis factoring (PAF) set as the extraction method due to the skewness of the data [36] and eigen types based on exploratory factor analysis (EFA).

Exploratory structural equation modelling (ESEM) can be used to investigate the structure of factors, and does so by integrating features of EFA and confirmatory factor analysis (CFA) [37]. ESEM builds on these techniques by permitting the specification of the expected factor number, which in this study is based on findings from the Hull method and theoretical consideration, while allowing all cross-loadings (i.e. items can have non-zero loadings across factors, which is inherent in psychological measurement) [38]. ESEM was run with robust maximum likelihood and geomin rotation using the 'esem' function in package 'lavaan'. Items with factor loadings $<0.40$ were omitted if there was no theoretical justification for retaining them.

Item response theory is a framework for model-based measurement of items, whereby responses are modelled as a function of individual respondent characteristics and item properties [39]. While traditional item response theory is limited by the assumption of unidimensionality (i.e. that there is one dominant latent trait being measured and that this drives the responses observed for each item in the measure), multidimensional item response theory (MIRT) extends both unidimensional item response theory and factor analysis, enabling the analysis of multiple constructs simultaneously [40]. We ran a MIRT graded response model using flexMIRT software [41] which is used for polytomous responses such as Likert scales [42], to examine the extent to which the items differentiate among individuals across different degrees of the underlying traits. The slope parameter (a) refers to the item's discriminative ability, with higher values indicating a stronger association with the construct [43]. Items with a slope parameter (a) between 1.35 and 1.70 were considered to have high discrimination and above 1.70 considered to have very high discrimination [44]. The information from these analyses and theory were combined to select a final pool of items for each of the factors.

\section{Validation of the Factor Structure}

Once the number of factors and item selection were established using the calibration samples of waves 17 and 19, we crossvalidated the factor structure using CFA in the validation samples of both waves. CFA tests whether the data fit the hypothesised factor structure by constraining the correlations between factors to zero. Model fit measures are then obtained to assess how well the proposed model captures the covariance between all the items in the model, with comparative fit index (CFI) $>0.90$, root mean square error of approximation (RMSEA) $<0.08$ and standardized root mean square residual (SRMR) $<0.08$ indicative of good fit [45]. The CFA was run using the 'lavaan' package with maximum likelihood robust (MLR) to address non-normality.

\section{Measurement Invariance of the Adapted Scales}

We evaluated measurement invariance of the scale across time (waves 17 and 19), gender and age groups. In a stepwise process, we tested configural (i.e., whether the factor structure was the same across groups), metric (i.e., whether factor loadings were the same across groups), scalar (whether the mean structures/intercepts of items were the same across groups) and strict invariance (i.e., whether the residual error was the same across groups). Configural invariance was established with a CFI $>0.90$ and RMSEA $<0.06$. Metric, scalar and strict invariance were established when $\Delta$ CFI was $<0.01$ and when $\Delta \chi^{2}$ was non-significant $(p>0.05)[46]$. Partial metric and/or scalar measurement invariance was established when at least two factor loadings and/or intercepts per latent factor were invariant.

\section{Internal Consistency of the Adapted Scales}

We tested the internal consistency of the identified scales by generating Cronbach's alpha (a) and McDonald's omega ( $\omega)$ using the 'psych' package. While Cronbach's a is the more widely used measure of internal consistency, it is based on the assumption of tau-

Page $4 / 15$ 
equivalence i.e., that all items have equal factor loadings. McDonald's $\omega$, instead, has the advantage of accounting for the strength of association between items by assuming a congeneric model, i.e., factor loadings may differ between items. This corrects for underand overestimations from Cronbach's a [47]. Recommended cut-offs for $a$ and $\omega$ are the same, with coefficients between 0.60 and 0.70 considered acceptable, and 0.70 or higher as good [48].

\section{Construct Validity: The Association of the Adapted Scales with Psychological Distress and Mental Well-being}

To assess construct validity, we examined Spearman's rank order correlations between the adapted scales with the K10 and MCS, respectively. To assess the performance of the scales in discriminating differences in psychological distress and poor mental health, using the K10 threshold of 22 or higher and MCS score of 42 or less, respectively, we then examined the area under the curve (AUC) generated by non-parametric Receiver Operating Characteristic (ROC) analysis in SPSS. Based on examination of item scores within scales and theoretical justification, we developed a cut-off score to classify individuals within each scale as expressing that trait or not. We conducted univariable logistic regression to identify the odds of an individual having psychological distress or poor mental health if they expressed the trait.

\section{Results}

\section{Sample Characteristics}

There were 15,637 respondents with complete data for the 10 social support items included in the HILDA questionnaire in wave 17 and 15,693 in wave 19. As shown in Table 1, sample characteristics were similar across each wave. The average age of respondents was 46 years, $47 \%$ were male, $10 \%$ spoke a language other than English and $30 \%$ had a long-term health condition. 


\begin{tabular}{|c|c|c|}
\hline Item & Wave $17(\mathrm{~N}=15,637)$ & Wave $19(\mathrm{~N}=15,693)$ \\
\hline Age, $M(\mathrm{SD})$ & $45.5(18.9)$ & $46.1(19.1)$ \\
\hline Male, $n(\%)$ & $7355(47.0)$ & $7370(47.0)$ \\
\hline Speak language other than English, $n(\%)^{\square}$ & $1569(10.0)$ & $1544(9.8)$ \\
\hline Long term health condition, $n(\%)^{\square}$ & $4670(29.9)$ & $4586(29.2)$ \\
\hline \multicolumn{3}{|l|}{ Marital status, $n(\%)^{\square}$} \\
\hline Married/de facto & $10055(64.3)$ & $9398(59.9)$ \\
\hline Never married & $3577(22.9)$ & $3589(22.9)$ \\
\hline Divorced/separated & $1353(8.7)$ & $1984(12.6)$ \\
\hline Widowed & $651(4.2)$ & $722(4.6)$ \\
\hline \multicolumn{3}{|l|}{ Employment status, $n(\%)^{\square}$} \\
\hline Full time & $6649(42.5)$ & $6702(42.8)$ \\
\hline Part time & $3267(20.9)$ & $3246(20.7)$ \\
\hline Retired & 3061 (19.6) & $3213(20.5)$ \\
\hline Unemployed/other & $2639(16.9)$ & $2513(16.0)$ \\
\hline \multicolumn{3}{|l|}{ Household structure, $n(\%)$} \\
\hline Lone person & $2421(15.5)$ & $2477(15.8)$ \\
\hline Lone person with child & $433(2.8)$ & $426(2.7)$ \\
\hline Couple with child & $3642(23.3)$ & $3607(23.0)$ \\
\hline Couple without child & 4985 (31.9) & $5078(32.4)$ \\
\hline Other & $4156(26.6)$ & $4105(26.2)$ \\
\hline \multicolumn{3}{|l|}{ SEIFA quintile, $n(\%)^{\square}$} \\
\hline 1-2 (most disadvantaged) & $2928(18.7)$ & 2907 (18.5) \\
\hline $3-4$ & 3105 (19.9) & 3072 (19.6) \\
\hline $5-6$ & 3058 (19.6) & $3132(20.0)$ \\
\hline 7-8 & 3270 (20.9) & $3309(21.1)$ \\
\hline 9-10 (least disadvantaged) & 3271 (20.9) & $3261(20.8)$ \\
\hline SF-36 MCS score, $M(\mathrm{SD})^{\square}$ & $48.0(10.9)$ & $47.3(11.3)$ \\
\hline SF-36 MCS score $\leq 42, n(\%)^{\square}$ & $3673(24.2)$ & $4043(26.3)$ \\
\hline Psychological distress (K10) score, $M(\mathrm{SD})^{\square}$ & $16.4(6.9)$ & $16.8(7.2)$ \\
\hline Psychological distress (K10) score $\geq 22, n(\%)^{\square}$ & 2895 (18.5) & $3151(20.1)$ \\
\hline \multicolumn{3}{|l|}{${ }^{\square}$ missing data } \\
\hline $\begin{array}{l}\text { SEIFA, Socio-Economic Indexes for Areas; MCS, } \\
\text { distress; MCS } \leq 42 \text { corresponds to poor mental }\end{array}$ & Summary; K10 score & ponds to psychologica \\
\hline
\end{tabular}


The Hull method indicated that a model with three factors provided the best fit in both waves 17 and 19 (Supplementary Figures 2a and 2b). Therefore, we fitted a three-factor model using ESEM and a MIRT model with three dimensions to select items. Factor loadings and model fit statistics from the ESEM in waves 17 and 19 are provided in Table 2, as are results from the MIRT graded response model with three dimensions in wave 19. The MIRT model indicated that all items had high discrimination on their respective factor in both waves.

Table 2

Item loadings and fit statistics from ESEM and MIRT models with three factors

\begin{tabular}{|c|c|c|c|c|c|c|c|c|c|}
\hline \multirow[b]{3}{*}{ Item } & \multicolumn{3}{|c|}{ Wave 17} & \multicolumn{6}{|c|}{ Wave 19} \\
\hline & \multicolumn{3}{|l|}{ ESEM } & \multicolumn{3}{|c|}{ ESEM } & \multicolumn{3}{|l|}{ MIRT } \\
\hline & $\begin{array}{l}\mathrm{F} 1 \\
(\lambda)\end{array}$ & $\begin{array}{l}\mathrm{F} 2 \\
(\lambda)\end{array}$ & $\begin{array}{l}\text { F3 } \\
(\lambda)\end{array}$ & $\begin{array}{l}\mathrm{F} 1 \\
(\lambda)\end{array}$ & $\begin{array}{l}\mathrm{F} 2 \\
(\lambda)\end{array}$ & $\begin{array}{l}\text { F3 } \\
(\lambda)\end{array}$ & a1 & a2 & a3 \\
\hline People don't come to visit me as often as I would like & 1.15 & -0.06 & 0.04 & 1.16 & -0.07 & 0.02 & 1.28 & 0.00 & 0.00 \\
\hline I often need help from other people but can't get it & 0.86 & 0.14 & 0.32 & 0.89 & 0.13 & 0.30 & 1.64 & 0.00 & 0.00 \\
\hline I don't have anyone that I can confide in & 0.38 & 0.17 & 1.01 & 0.17 & 0.06 & 1.30 & 0.00 & 2.24 & 0.00 \\
\hline I have no one to lean on in times of trouble & 0.03 & 0.01 & 1.61 & 0.08 & 0.03 & 1.38 & 0.00 & 3.75 & 0.00 \\
\hline $\begin{array}{l}\text { There is someone who can always cheer me up when I'm } \\
\text { down }\end{array}$ & 0.36 & 0.77 & 0.18 & 0.29 & 0.82 & 0.24 & 0.00 & 0.00 & 1.40 \\
\hline I often feel very lonely & 0.98 & 0.19 & 0.29 & 0.85 & 0.19 & 0.43 & 1.40 & 0.00 & 0.00 \\
\hline $\begin{array}{l}\text { I enjoy the time I spend with the people who are } \\
\text { important to me }\end{array}$ & -0.01 & 0.76 & 0.07 & 0.01 & 0.78 & 0.01 & 0.00 & 0.00 & 1.52 \\
\hline $\begin{array}{l}\text { When something's on my mind, just talking with the } \\
\text { people I know can make me feel better }\end{array}$ & -0.02 & 1.15 & 0.04 & -0.01 & 1.15 & 0.07 & 0.00 & 0.00 & 1.98 \\
\hline $\begin{array}{l}\text { When I need someone to help me out, I can usually find } \\
\text { someone }\end{array}$ & 0.46 & 0.92 & 0.19 & 0.46 & 0.90 & 0.22 & 0.00 & 0.00 & 2.65 \\
\hline Chi-square & \multicolumn{3}{|c|}{234.63} & \multicolumn{3}{|c|}{243.63} & \multicolumn{3}{|l|}{-} \\
\hline $\mathrm{CFI}$ & \multicolumn{3}{|l|}{0.992} & \multicolumn{6}{|c|}{0.992} \\
\hline TLI & \multicolumn{3}{|l|}{0.977} & \multicolumn{6}{|c|}{0.975} \\
\hline RMSEA (90\% Cls) & \multicolumn{3}{|c|}{$0.049(0.043,0.054)$} & \multicolumn{3}{|c|}{$0.051(0.045,0.056)$} & \multicolumn{3}{|l|}{-} \\
\hline SRMR & \multicolumn{3}{|l|}{0.013} & \multicolumn{3}{|c|}{0.012} & & & \\
\hline
\end{tabular}

Three items (item 1: "People don't come to visit me as often as I would like", item 2: "I often need help from other people but can't get $i t$ " and item 7: "I often feel very lonely") had the strongest loadings on one factor, reflecting the concept of loneliness.

The second factor included four items reflecting social isolation, including item 6: "There is someone who can always cheer me up when I'm down", item 8: "I enjoy the time I spend with the people who are important to me", item 9: "When something's on my mind, just talking with the people I know can make me feel better" and item 10: "When I need someone to help me out, I can usually find someone". The items included in the loneliness and social isolation sub-scales are summarized in Supplementary Table 1.

Items 4: "I don't have anyone that I can confide in" and 5: "I have no one to lean on in times of trouble" distinctly loaded onto a third factor. The use of two items to identify an underlying construct has been recognized as problematic, with true-score theory indicating that more items lead to better construct validity and reliability $[49,50]$. Analysis of this two-item sub-scale was therefore discontinued. 
Item 3: "I seem to have lots of friends" was also omitted from further analyses due to low loadings (0.38) and theoretically not reflecting the concept of loneliness or social support.

\section{Validation of Factor Structure}

CFA results indicated good fit of the three-factor model, with a CFI of 0.953 in wave 17 and 0.955 in wave 19, and a SRMR of 0.050 in wave 17 and 0.048 in wave 19. Model fit statistics for the CFA are also provided in Table 3.

Table 3

Fit statistics from the CFA model with three factors

\begin{tabular}{|lll|}
\hline & Wave 17 & Wave 19 \\
\hline Chi-square & 1320.78 & 1267.92 \\
\hline CFI & 0.953 & 0.955 \\
\hline TLI & 0.929 & 0.932 \\
\hline RMSEA (Cls) & $0.083(0.080,0.087)$ & $0.082(0.078,0.086)$ \\
\hline SRMR & 0.050 & 0.048 \\
\hline
\end{tabular}

\section{Measurement Invariance}

Measurement invariance for the three-factor scale across waves, gender and age are shown in Table 4. Results showed evidence for full configural, metric, scalar and strict invariance across waves 17 and 19. In Wave 19, we found evidence for configural and partial scalar invariance across gender. There was also evidence for configural and partial metric invariance across age. 
Table 4

Measurement invariance for the three-factor model across waves and gender

\begin{tabular}{|c|c|c|c|c|c|c|c|c|c|c|c|}
\hline & AIC & aBIC & $x^{2}$ & $\Delta \chi^{2}$ & $\begin{array}{l}\mathrm{p}- \\
\text { value }\end{array}$ & $\mathrm{CFI}$ & $\Delta \mathrm{CFI}$ & RMSEA & $\triangle \mathrm{RMSEA}$ & SRMR & $\triangle \mathrm{SRMR}$ \\
\hline \multicolumn{12}{|l|}{$\begin{array}{l}\text { Across } \\
\text { waves (17 } \\
\text { and 19) }\end{array}$} \\
\hline $\begin{array}{l}\text { Configural } \\
\text { invariance }\end{array}$ & 933079.20 & 933399.13 & 4931.2 & & & 0.95 & & 0.07 & & 0.04 & \\
\hline $\begin{array}{l}\text { Metric } \\
\text { invariance }\end{array}$ & 933074.19 & 933363.16 & 4938.2 & 7.0 & 0.60 & 0.95 & 0.00 & 0.06 & -0.01 & 0.04 & 0.00 \\
\hline $\begin{array}{l}\text { Scalar } \\
\text { invariance }\end{array}$ & 933068.25 & 933326.27 & 4944.2 & 6.0 & 0.42 & 0.95 & 0.00 & 0.06 & 0.00 & 0.04 & 0.00 \\
\hline $\begin{array}{l}\text { Strict } \\
\text { invariance }\end{array}$ & 933069.55 & 933281.12 & 4963.5 & 19.3 & 0.58 & 0.95 & 0.00 & 0.06 & 0.00 & 0.04 & 0.00 \\
\hline \multicolumn{12}{|l|}{$\begin{array}{l}\text { Across } \\
\text { sex in } \\
\text { Wave } 19 \\
\text { (male and } \\
\text { female) }\end{array}$} \\
\hline $\begin{array}{l}\text { Configural } \\
\text { invariance }\end{array}$ & 427284.77 & 427556.50 & 2353.1 & & & 0.95 & & 0.07 & & 0.04 & \\
\hline $\begin{array}{l}\text { Metric } \\
\text { invariance }\end{array}$ & 427332.53 & 427577.96 & 2412.9 & 59.8 & $<0.001$ & 0.94 & -0.01 & 0.07 & 0.00 & 0.05 & 0.01 \\
\hline $\begin{array}{l}\text { Partial } \\
\text { metric } \\
\text { invariance }\end{array}$ & 427383.94 & 427642.52 & 2458.3 & 45.4 & $<0.001$ & 0.94 & 0.00 & 0.07 & 0.00 & 0.05 & 0.00 \\
\hline $\begin{array}{l}\text { Scalar } \\
\text { invariance }\end{array}$ & 427597.94 & 427817.08 & 2690.3 & 232.0 & $<0.001$ & 0.94 & 0.00 & 0.07 & 0.00 & 0.05 & 0.00 \\
\hline $\begin{array}{l}\text { Partial } \\
\text { scalar } \\
\text { invariance }\end{array}$ & 427343.91 & 427576.19 & 2413.3 & 0.4 & 0.79 & 0.94 & 0.00 & 0.07 & 0.00 & 0.05 & 0.00 \\
\hline $\begin{array}{l}\text { Strict } \\
\text { invariance }\end{array}$ & 427769.40 & 427949.09 & 2879.8 & 466.5 & $<0.001$ & 0.94 & 0.00 & 0.06 & 0.01 & 0.07 & 0.02 \\
\hline \multicolumn{12}{|l|}{$\begin{array}{l}\text { Across } \\
\text { age } \\
\text { categories }\end{array}$} \\
\hline $\begin{array}{l}\text { Configural } \\
\text { invariance }\end{array}$ & 459166.47 & 459834.67 & 2858.5 & & & 0.95 & & 0.07 & & 0.05 & \\
\hline $\begin{array}{l}\text { Metric } \\
\text { invariance }\end{array}$ & 459288.70 & 459849.99 & 3028.7 & 170.2 & $<0.001$ & 0.95 & 0.00 & 0.07 & 0.00 & 0.05 & 0.00 \\
\hline $\begin{array}{l}\text { Partial } \\
\text { metric } \\
\text { invariance }\end{array}$ & 459170.39 & 459802.96 & 2869.0 & 10.5 & 0.08 & 0.94 & -0.01 & 0.07 & 0.00 & 0.05 & 0.00 \\
\hline $\begin{array}{l}\text { Scalar } \\
\text { invariance }\end{array}$ & 459616.90 & 460071.27 & 3404.9 & 535.9 & $<0.001$ & 0.94 & 0.00 & 0.07 & 0.00 & 0.06 & 0.01 \\
\hline $\begin{array}{l}\text { Partial } \\
\text { scalar } \\
\text { invariance }\end{array}$ & 460232.25 & 460472.81 & 2602.2 & -266.8 & $<0.001$ & 0.94 & 0.00 & 0.07 & 0.00 & 0.06 & 0.00 \\
\hline $\begin{array}{l}\text { Strict } \\
\text { invariance }\end{array}$ & 460800.67 & 460983.31 & 3196.6 & 594.4 & $<0.001$ & 0.94 & 0.00 & 0.07 & 0.00 & 0.06 & 0.00 \\
\hline
\end{tabular}




\section{Internal Consistency}

The Cronbach's $a$ and McDonald's $\omega$ of the loneliness and social isolation scales in each of waves 17 and 19 are shown in Table 5. Across the two waves, both scales demonstrated good reliability, with the $\alpha$ and $\omega$ coefficients of 0.70 and 0.71 for the 3 -item loneliness scale, respectively, in both wave 17 and wave 19. The 4-item social isolation scale reported respective $\alpha$ and $\omega$ coefficients of 0.80 and 0.81 , respectively for both wave 17 and wave 19 .

Table 5

Internal consistency of the loneliness and social isolation scales

\begin{tabular}{|lllll|}
\hline & \multicolumn{2}{l}{ Loneliness 3-item } & \multicolumn{2}{c|}{ Social Isolation 4-item } \\
\hline & Wave 17 & Wave 19 & Wave 17 & Wave 19 \\
\hline Cronbach's a & 0.70 & 0.70 & 0.80 & 0.80 \\
\hline McDonald's $\omega$ & 0.71 & 0.71 & 0.81 & 0.81 \\
\hline
\end{tabular}

\section{Construct Validity}

The loneliness and social isolation scales were correlated significantly with the K10 and MCS. We saw a positive correlation with the loneliness sub-scale scale and K10, with correlation coefficients of 0.48 across the two waves. We found a negative correlation between the loneliness sub-scale and the MCS, with a consistent coefficient of -0.46 . In contrast, the positively worded social isolation scale correlated negatively with the K10 and positively with the MCS, with coefficients of -0.37 and 0.38 , respectively (Table 6).

Table 6

Spearman's correlations, area under the curve and odds ratios for loneliness and social isolation scales

\begin{tabular}{|c|c|c|c|c|}
\hline & \multicolumn{2}{|l|}{ Wave 17} & \multicolumn{2}{|l|}{ Wave 19} \\
\hline & K10 & MCS & K10 & MCS \\
\hline \multicolumn{5}{|l|}{ Spearman's correlations ${ }^{a}$} \\
\hline Loneliness & 0.48 & -0.46 & 0.48 & -0.46 \\
\hline Social isolation & -0.37 & 0.38 & -0.38 & 0.38 \\
\hline \multicolumn{5}{|c|}{ Receiver operating characteristic: area under the curve (AUC) } \\
\hline Loneliness & 0.78 & 0.75 & 0.77 & 0.74 \\
\hline Social isolation & 0.73 & 0.70 & 0.73 & 0.70 \\
\hline \multicolumn{5}{|c|}{ Odds ratios - logistic regression $(95 \% \mathrm{Cls})^{\mathrm{a}, \mathrm{b}}$} \\
\hline \multirow[t]{2}{*}{ Loneliness (cut-off >4) } & 6.14 & 5.57 & 5.60 & 5.07 \\
\hline & $(5.58-6.75)$ & $(5.07-6.12)$ & $(5.10-6.14)$ & $(4.62-5.55)$ \\
\hline \multirow[t]{2}{*}{ Social isolation (cut-off $<4$ ) } & 4.67 & 3.99 & 4.74 & 4.24 \\
\hline & $(4.09-5.33)$ & $(3.49-4.56)$ & $(4.15-5.42)$ & $(3.70-4.85)$ \\
\hline \multicolumn{5}{|c|}{${ }^{\mathrm{a}}$ All significant at the 0.01 level } \\
\hline
\end{tabular}


The AUC for the loneliness scale showed fair performance, with areas ranging from 0.77 to 0.78 against the K10 psychological distress scores, and from 0.74 to 0.75 for the MCS. Similarly, the AUC for the social isolation scale was consistently fair against the $\mathrm{K} 10$ and MCS, with coefficients of 0.73 and 0.70 , respectively (Table 6).

The threshold for classification of loneliness was determined to be a median item score of less than 4 , and for social isolation a median item score of greater than 4 , as this represents having a majority level of agreement (for loneliness items) or disagreement (for social isolation items).

The results of the univariable logistic regression to assess the relationship between dichotomous loneliness and social isolation variables and the categorical indicators of psychological distress and poor mental health (from the K10 and MCS), are shown in Table 6 . Using the threshold median score for loneliness of less than 4 , we found that $15 \%$ of respondents were lonely in waves 17 and 19. For social isolation, a threshold median score of greater than 4 resulted in $6 \%$ of respondents being classified as socially isolated in each of the waves. Across the two waves, we found that individuals classified as lonely were approximately six times more likely to be psychologically distressed than non-lonely participants, and 5 times more likely to have poor mental health. Similarly, those classified as socially isolated were approximately 4.5 times more likely to be psychologically distressed than those not socially isolated, and four times more likely to have poor mental health.

\section{Discussion}

In this report, we identified measures for loneliness and social isolation from 10 items used to measure social support in a large, population-based cohort study. The loneliness and social isolation scales demonstrated good measurement invariance, reliability and construct validity when compared with measures for psychological distress and mental health.

We found that the first factor reflecting loneliness contained negatively worded items, which is a pattern previously observed in psychometric analyses of loneliness scales [51]. However, the three items in the loneliness scale are consistent with previous theory and literature on loneliness, which recognises it as the subjective evaluation of the current state of their relationships. Social isolation, related to the amount of contact a person has with others, is reflected in the four-item scale of the second factor which assesses the availability and utilisation of social relationships [52]. Although the social isolation sub-scale items were found to be all positively worded, not all the positive items possessed sufficient loading to be included in the second component (i.e., item 3 was omitted due to low loadings). While it is possible that these scales were subject to acquiescence bias, the participants in the HILDA survey completed the 10 items together (mix of positively and negatively worded items), which is a known method for controlling the bias [53]. Further, not all the negatively worded items loaded together, with items 4 and 5 loading onto their own factor separate to the other negatively worded items.

Investigation of measurement invariance found agreement of the scales across waves. Between males and females, partial scalar invariance was found. Females had higher intercepts (means) for item 9: "When something's on my mind, just talking with the people I know can make me feel better", whereas males had higher intercepts for item 7: "I often feel very lonely" and item 10: "When I need someone to help me out, I can usually find someone". This suggests that while it is possible to compare the latent structure, latent mean levels and relationships between variables, caution should be used when comparing the observed means for the scales [54].

Inclusion of the K10 psychological distress scale and MCS derived from the SF-36 in the HILDA survey enabled comparisons between these and the loneliness and social isolation scales. We found that loneliness and social isolation were consistently and significantly correlated with psychological distress and poor mental health across the waves, and that loneliness was a slightly better predictor of these outcomes than social isolation. Recent studies have similarly shown that loneliness is associated with a greater risk of mental health problems and severe depression compared to other similar constructs such as social support [55-57]. This consistency with other studies further supports the validity of the scales as measures of their respective constructs.

Given the significant impact of loneliness and social isolation on mental and physical health, population level monitoring is needed to be able to assess trends in these important social conditions, identify priority groups, and monitor the impact of policies and programs [4]. To achieve this, the scales need to be practical and ideally be able to generate prevalence estimates for those at greatest risk. The Government of the United Kingdom has played a leading role in loneliness research, policy and monitoring, internationally, and has recognised that inconsistent measures reduce the ability to compare data and limits our understanding of 
these critical issues [58]. Consistent measures for loneliness and social isolation are needed across different research areas, including population studies, in order to identify and monitor these critical social, community and public health problems [59].

The ongoing COVID-19 pandemic has also placed a spotlight on the need for population-level monitoring of loneliness and isolation, as social interactions have been restricted due to regulations around physical and social distancing, self-isolation and quarantining [60]. Recent evidence from the UK and USA suggests the prevalence of isolation and loneliness was high during COVID-19, particularly in the acute phase of the outbreak [61, 62]. However, the different measures used, lack of accurate pre-COVID loneliness data and lack of data from other affected countries has hindered our ability to assess the impact of COVID-19 on loneliness and isolation.

This study has a number of strengths, including the robust examination of the psychometric properties of items completed by over 15,000 participants in two waves of the HILDA study. The large sample size enabled us to determine the number of factors and items using recommended analytical techniques including the Hull method, which has shown to outperform traditional methods of factor analysis [35]. We then demonstrated the robustness of our findings through cross-validation in another sample using CFA and by testing longitudinal and cross-sectional measurement invariance. Inclusion of widely used measures of mental health and psychological distress in the HILDA survey permitted rigorous validation of the loneliness and social isolation measures. However, this study has some limitations. First, the survey was conducted in English only, with participants tending to have higher socioeconomic status. We were unable to test the sensitivity and responsiveness of the measures, so their suitability as intervention evaluation measures is not known. While this study included a large sample from a nationally representative survey, further validation will be required for use in other countries.

\section{Conclusions}

The growing attention to loneliness and social isolation worldwide, and their known associations with morbidity and mortality [63, 64], calls for improved tools to measure the prevalence and burden of these conditions. The present study provides an important contribution by identifying and assessing the psychometric properties of two short self-report scales for loneliness and social isolation in Australia. Our findings demonstrate that these scales provide valid and reliable measures for loneliness and social isolation that are suitable for use in population surveys to improve future research and population monitoring.

\section{Declarations}

\section{Ethics approval and consent to participate}

This project was granted exemption from ethics review from The University of Sydney Human Research Ethics Committee.

\section{Consent for publication}

Not applicable.

\section{Availability of data and materials}

The dataset supporting the conclusions of this article are available to researchers living in Australia or overseas through the National Centre for Longitudinal Data Dataverse. Information about applying for access to the data is available at: https://dataverse.ada.edu.au/dataverse/ncld.

\section{Competing interests}

The authors declare that they have no competing interests.

\section{Funding}

No funding was provided for this study.

\section{Authors' contributions}


KEM contributed to writing, methodology and formal analysis; BJS contributed to study conceptualisation, supervision, methodology and editing; KBO contributed to methodology, formal analysis, investigation and data curation; PP contributed to supervision, writing, reviewing \& editing; MHL contributed to study conceptualisation, supervision, and editing. All authors read and approved the final manuscript.

\section{Acknowledgements}

The authors would like to thank Sherry Vasan for her contribution in referencing this manuscript.

\section{References}

1. Peplau, L. and D. Perlman, Perspectives on loneliness, in Loneliness: A Sourcebook of Current Theory, Research and Therapy., L. Peplau and D. Perlman, Editors. 1982, John Wiley and Sons.: New York. p. 1-20.

2. Valtorta, N. and B. Hanratty, Loneliness, isolation and the health of older adults: do we need a new research agenda? J Royal Soc Med. 2012; 105(12): 518-522.

3. Holt-Lunstad, J., et al., Loneliness and social isolation as risk factors for mortality: a meta-analytic review. Perspectives on Psychological Science. 2015; 10(2): 227-237.

4. Lim, M.H., R. Eres, and S. Vasan, Understanding loneliness in the twenty-first century: an update on correlates, risk factors, and potential solutions. Soc Psychiatry Psychiatr Epidemiol. 2020; 55: 793-810.

5. Sinickas, A., Finding A Cure For Survey Fatigue. Strategic Communication Management. 2007; 11(2): 11.

6. Ziegler, M., C.J. Kemper, and P. Kruyen, Short scales - Five misunderstandings and ways to overcome them. Journal of Individual Differences. 2014; 35(4): 185-189.

7. Valtorta, N., et al., Loneliness, social isolation and social relationships: what are we measuring? A novel framework for classifying and comparing tools. BMJ Open. 2016; 6(4): e010799.

8. Veazie, S., et al., Addressing Social Isolation To Improve the Health of Older Adults: A Rapid Review. 2019, Agency for Healthcare Research and Quality (US): Rockville (MD).

9. Russell, UCLA Loneliness Scale (Version 3): Reliability, Validity, and Factor Structure. J Pers Assess. 1996; 66(1): 20-40.

10. De Jong Gierveld, J. and F. Kamphuls, The Development of a Rasch-Type Loneliness Scale. Appl Psychol Meas. 1985; 9(3): 289299.

11. Lubben, J.E., Assessing social networks among elderly populations. Fam Community Health. 1988; 11(3).

12. Berkman, L. and L. Breslow, Health and ways of living. 1983, New York: Oxford University Press.

13. Hughes, M.E., et al., A Short Scale for Measuring Loneliness in Large Surveys: Results From Two Population-Based Studies. Res Aging. 2004; 26(6): 655-672.

14. De Jong Gierveld, J. and T.V. Tilburg, A 6-Item Scale for Overall, Emotional, and Social Loneliness:Confirmatory Tests on Survey Data. Res Aging. 2006; 28(5): 582-598.

15. Lubben, J., et al., Performance of an Abbreviated Version of the Lubben Social Network Scale Among Three European Community-Dwelling Older Adult Populations. Gerontologist. 2006; 46(4): 503-513.

16. Wilkins, R., et al., The Household, Income and Labour Dynamics in Australia Survey: Selected Findings from Waves 1 to 17. 2019 : Melbourne Institute: Applied Economic \& Social Research, University of Melbourne.

17. Butterworth, P. and T. Crosier, The validity of the SF-36 in an Australian National Household Survey: demonstrating the applicability of the Household Income and Labour Dynamics in Australia (HILDA) Survey to examination of health inequalities. BMC Public Health. 2004; 4(1): 44.

18. Luchetti, M., et al., Loneliness is associated with risk of cognitive impairment in the Survey of Health, Ageing and Retirement in Europe. Int J Geriatr Psychiatry. 2020; 35(7): 794-801.

19. Stickley, A. and A. Koyanagi, Loneliness, common mental disorders and suicidal behavior: Findings from a general population survey. J Affect Disord. 2016; 197: 81-87.

20. Lau, S. and G.E. Gruen, The Social Stigma of Loneliness: Effect of Target Person's and Perceiver's Sex. Pers Soc Psychol Bull. 1992; 18(2): 182-189. 
21. Dahlberg, L., et al., Predictors of loneliness among older women and men in Sweden: A national longitudinal study. Aging Ment Health. 2015; 19(5): 409-417.

22. Nicolaisen, M. and K. Thorsen, Who are Lonely? Loneliness in Different Age Groups (18-81 Years Old), Using Two Measures of Loneliness. Int J Aging Hum Dev. 2014; 78(3): 229-257.

23. Victor, C.R., V. Burholt, and W. Martin, Loneliness and Ethnic Minority Elders in Great Britain: An Exploratory Study. J Cross Cult Gerontol. 2012; 27(1): 65-78.

24. Loo, R., A caveat on using single-item versus multiple-item scales. J Manag Psychol. 2002; 17(1): 68-75.

25. Henderson, S., et al., The Patient's Primary Group. Br J Psychiatry. 1978; 132(1): 74-86.

26. Marshall, N.L. and R.C. Barnett, Work-family strains and gains among two-earner couples. J Community Psychol. 1993; 21(1): 6478.

27. Smith, N. and D. Weatherburn, Personal stress, financial stress, social support and women's experiences of physical violence: A Iongitudinal analysis, in Crime and Justice. 2013, NSW Bureau of Crime Statistics and Research: Sydney, Australia.

28. Flood, M., Mapping Loneliness in Australia. Discussion Paper Number 76. ed. T.A. Institute. 2005, Canberra, Australia.

29. Ware and C.D. Sherbourne, The MOS 36-item short-form health survey (SF-36). I. Conceptual framework and item selection. Med Care. 1992; 30(6): 473-83.

30. Australian Bureau of Statistics, National Health Survey: SF-36 population norms. 1995: Canberra, Australia.

31. Ware, J.E., SF-36 Physical and Mental Health Summary Scales: A User's Manual. 1994: Health Institute, New England Medical Center.

32. Kessler, R.C., et al., Short screening scales to monitor population prevalences and trends in non-specific psychological distress. Psychol Med. 2002; 32(6): 959-76.

33. Australian Bureau of Statistics, National Health Survey: users' guide 2017-18. ABS cat. no. 4364.0. 2019: Canberra, Australia.

34. Andrews, G. and T. Slade, Interpreting scores on the Kessler Psychological Distress Scale (K10). Aust N Z J Public Health. 2001; 25(6): 494-497.

35. Lorenzo-Seva, U., M. Timmerman, and H. Kiers, The Hull Method for Selecting the Number of Common Factors. Multivariate Behav Res. 2011; 46: 340-364.

36. Costello, A.B. and J. Osborne, Best Practices in Exploratory Factor Analysis: Four Recommendations for Getting the Most From Your Analysis. Pract Assess Res Evaluation. 2005; 10: 1-9.

37. Marsh, H.W., et al., Exploratory Structural Equation Modeling: An Integration of the Best Features of Exploratory and Confirmatory Factor Analysis. Annu Rev Clin Psychol. 2014; 10(1): 85-110.

38. Asparouhov, T. and B. Muthén, Exploratory Structural Equation Modeling. Struct Equ Model. 2009; 16(3): 397-438.

39. Reckase, M.D., Multidimensional Item Response Theory. 1 ed. Statistics for Social and Behavioral Sciences. 2009: SpringerVerlag New York.

40. Chalmers, R., Mirt: A Multidimensional Item Response Theory Package for the R Environment. J Stat Softw. 2012; 48(6).

41. Houts, C.R. and L. Cai, flexMIRT: Flexible Multilevel Multidimensional Item Analysis and Test Scoring. 2013, Chapel Hill, NC: Vector Psychometric Group.

42. Penfield, R.D., An NCME Instructional Module on Polytomous Item Response Theory Models. Educ Meas. 2014; 33(1): 36-48.

43. Lameijer, C.M., et al., Graded response model fit, measurement invariance and (comparative) precision of the Dutch-Flemish PROMIS ${ }^{\circledR}$ Upper Extremity V2.0 item bank in patients with upper extremity disorders. BMC Musculoskelet Disord. 2020; 21(1): 170.

44. Sharkness, J. and L. DeAngelo, Measuring Student Involvement: A Comparison of Classical Test Theory and Item Response Theory in the Construction of Scales from Student Surveys. Res High Educ. 2011; 52(5): 480-507.

45. Boateng, G.O., et al., Best Practices for Developing and Validating Scales for Health, Social, and Behavioral Research: A Primer. Front Public Health. 2018; 6: 149-149.

46. Chen, F.F., Sensitivity of Goodness of Fit Indexes to Lack of Measurement Invariance. Struct Equ Model. 2007; 14(3): $464-504$.

47. Trizano-Hermosilla, I. and J.M. Alvarado, Best Alternatives to Cronbach's Alpha Reliability in Realistic Conditions: Congeneric and Asymmetrical Measurements. Front Psychol. 2016; 7(769).

Page $14 / 15$ 
48. Leary, M.R., Introduction to Behavioral Research Methods. 2016: Pearson Education.

49. Eisinga, R., M.t. Grotenhuis, and B. Pelzer, The reliability of a two-item scale: Pearson, Cronbach, or Spearman-Brown? Int J Public Health. 2013; 58(4): 637-642.

50. Wainer, H. and D. Thissen, True score theory: The traditional method, in Test scoring. 2001, Lawrence Erlbaum Associates Publishers: Mahwah, NJ, US. p. 23-72.

51. Miller, T.R. and T.A. Cleary, Direction of Wording Effects in Balanced Scales. Educ Psychol Meas. 1993; 53(1): 51-60.

52. Fiordelli, M., et al., Differentiating objective and subjective dimensions of social isolation and apprasing their relations with physical and mental health in italian older adults. BMC Geriatr. 2020; 20: 472.

53. Friborg, O., M. Martinussen, and J.H. Rosenvinge, Likert-based vs. semantic differential-based scorings of positive psychological constructs: A psychometric comparison of two versions of a scale measuring resilience. Pers Individ Differ. 2006; 40(5): 873-884.

54. Steinmetz, H., Analyzing observed composite differences across groups: Is partial measurement invariance enough? Methodology. 2013; 9(1): 1-12.

55. Wang, J., et al., Associations between loneliness and perceived social support and outcomes of mental health problems: a systematic review. BMC Psychiatry. 2018; 18(1): 156.

56. Lim, M.H., et al., Loneliness over time: The crucial role of social anxiety. J Abnorm Psychol. 2016; 125(5): 620-630.

57. Beller, J. and A. Wagner, Disentangling Loneliness: Differential Effects of Subjective Loneliness, Network Quality, Network Size, and Living Alone on Physical, Mental, and Cognitive Health. J Aging Health. 2018; 30(4): 521-539.

58. HM Government, A connected society: A strategy for tackling loneliness, C. Department for Digital, Media and Sport, Editor. 2018: London, United Kingdom.

59. Ending Loneliness Together, Ending Loneliness Together in Australia White Paper. 2020: Australia.

60. Smith, B. and M. Lim, How the COVID-19 pandemic is focusing attention on loneliness and social isolation. Public Health Res Pract. 2020; 30(2): e3022008.

61. Luchetti, M., et al., The trajectory of loneliness in response to COVID-19. Am Psychol. 2020; 75(7): 897-908.

62. Groarke, J.M., et al., Loneliness in the UK during the COVID-19 pandemic: Cross-sectional results from the COVID-19 Psychological Wellbeing Study. PLOS ONE. 2020; 15(9): e0239698.

63. Perissinotto, C.M., I. Stijacic Cenzer, and K.E. Covinsky, Loneliness in older persons: a predictor of functional decline and death. Arch Intern Med. 2012; 172(14): 1078-1083.

64. Holt-Lunstad, J., et al., Loneliness and Social Isolation as Risk Factors for Mortality:A Meta-Analytic Review. Perspect Psychol Sci. 2015; 10(2): 227-237.

\section{Supplementary Files}

This is a list of supplementary files associated with this preprint. Click to download.

- HILDAsupplementarymaterial28092021.docx 